\title{
METHOD OF A PLANETARY ROVER LOCALIZATION BASED ON SYNTHETIC LUNOKHOD IMAGES
}

\author{
N.A. Kozlova ${ }^{\text {a }}$, A.E. Zubarev ${ }^{\text {a }}$, V.D. Patratiy ${ }^{\mathrm{a}}$, A.A. Konopikhin ${ }^{\mathrm{a}}$, J. Oberst $^{\mathrm{a}, \mathrm{b}, \mathrm{c}}$ \\ ${ }^{a}$ Moscow State University of Geodesy and Cartography, MIIGAiK Extraterrestrial laboratory (MExLab) 105064, Gorokhovsky \\ per.,4, Moscow, Russia - natally.ko@gmail.com \\ ${ }^{\mathrm{b}}$ German Aerospace Center (DLR), Germany \\ ${ }^{\mathrm{c}}$ Technical University Berlin, Germany
}

Commission IV, WG IV/8

KEY WORDS: Planetary Geodesy, Navigation, Photogrammetry, Software, the Moon, Lunokhod, Artificial image modelling.

\begin{abstract}
:
We present a method which is a continuation of work-in-progress paper (Kozlova et al., 2014). LRO NAC DEM, orthomosaic, and the results of detailed studies of the Lunokhod-2 route were used for modeling of individual sites in order to find observation points for archival panoramas. The method suggests determination of rover coordinates from photogrammetry processing of obtained surface images together with artificially modeled synthetic images produced from high-resolution DEM and orthomosaic. We have now tested the method on models and on real Lunokhod-2 data. The method proved its effectiveness on models, however, for Lunokhod-2 panoramas further research is needed to calibrate the camera properly.
\end{abstract}

\section{INTRODUCTION}

Russia has a long tradition in Moon exploration. Early highlights of lunar research include the first images of the Moon far side and the first soft landings. Also, the historic Lunokhods were the first planetary rovers, extremely successful in their early missions: Lunokhod-2 completed a traverse of about 40 $\mathrm{km}$ (Figure 1) and transferred about 100 panoramas of the lunar surface back to Earth. These data were used for operational decision-making during the missions, and subsequently for science and exploration purposes. Unfortunately, only small portions of the data have been processed and are available for the scientific community in digital form.

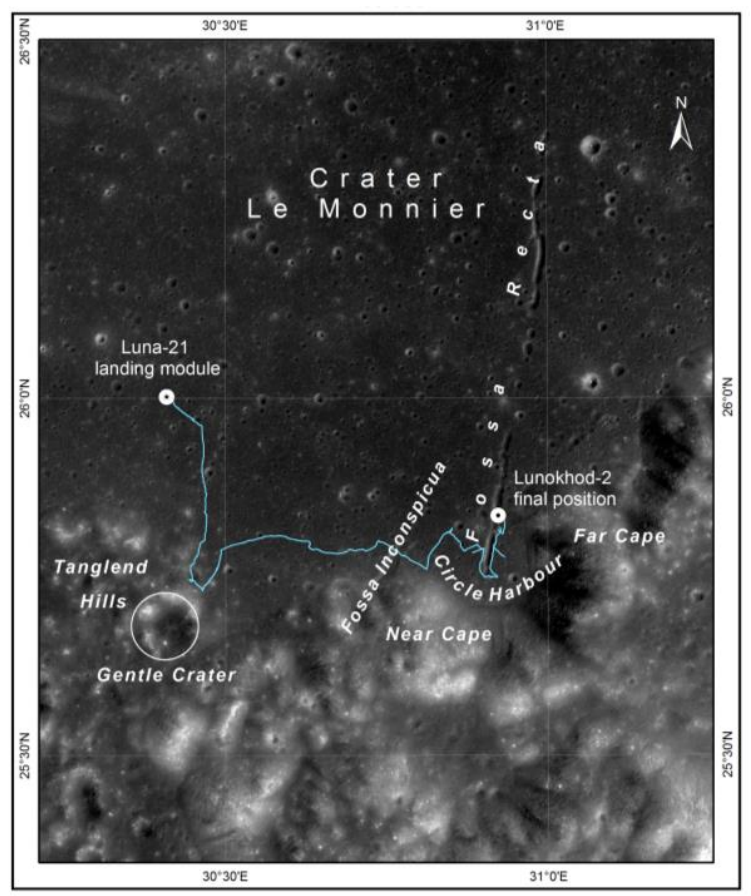

Figure 1. Map of Lunokhod-2 traverse
New plans in space research include going on with "Luna" program to study polar regions and even establish a lunar control point network by means of rovers which are capable of covering the distance of about $200 \mathrm{~km}$. During these missions a lot of experiments are planned (drilling, chemical analyses, etc.) so there is a need in a technique which would provide accurate binding of the investigation points to global Lunar coordinate system. In the same time it is better not to use additional equipment which will make the rover heavier and take lots of energy resources.

That is why, our idea is to use surface images obtained by the rover at points of investigation to help in determination of its coordinates. And we suggest that using specially modelled synthetic images as the base ones will make determinations easier and more accurate.

\section{METHOD, TECHNIQUE AND PRELIMINARY RESULTS}

So the goal is to identify the observation point of the image with the best accuracy. Then, as we know construction of the rover, we can define location of the rover itself or location of point of our experiment. The developed technique includes several steps:

- Preliminary orientation of the images. This step is necessary in order to find the exterior orientation for modeling of base images. In case the approximate coordinates and pointing are known, it is possible to use them. Otherwise, these elements are to be determined by visual comparison of the original surface image with a situation in the landing area. To do these one can use ancillary information about the route and all available maps, DEMs and orbital images. It is possible to use usual software for 3D-visualisation of DEM (such as ArcScene, Photomod, etc.) to help with the search of pointing direction. 
For now we are working mostly with Soviet Lunokhod data, so we carried out preliminary allocation of available panoramas along the identified rover wheel tracks (Kozlova et al., 2015). This work showed that very few surface images can be positioned steadily using GIS methods, as determination of coordinates of observation points for each panorama is complicated. By direct comparison with LRO NAC images it is possible to identify only several panoramas on remarkable places (e.g. near the Lander-module or which depicture prominent features). On other areas lunar relief seems very similar so other panoramas we had to put regularly onto the track between the identified ones. However, taking into account that Lunokhod-2 travelled during the 3rd lunar day up to 17.6 $\mathrm{km}$ but transmitted only 20 panoramas, it seems that uncertainty of their location in some cases can be up to several $\mathrm{km}$. We tried to determine the pointing azimuth by identifying hills which we can see on some of Lunokhod-2 panoramas and used ArcScene (http://www.esri.com) to visualize the horizon. It matches rather well with the horizon line seen on the part of a panorama. However, this program allows us to measure neither exact coordinates of observation point nor pointing direction. Also it does not support a model of Lunokhod camera. That is why visual identifications in GIS can be used only for preliminary orientation.

\begin{abstract}
- Modelling of synthetic base images. On this step the DEM is transformed into a synthetic surface image using preliminary exterior orientation and parameters of the camera for correct geometry of the image. Orbital images are used to form the texture of synthetic images (Figure 2). This step involves the use of a specialized module OrthoDEM2Cam (Zubarev et al., 2016) which calculates images that would be obtained by the rover standing at the selected place and looking in the selected pointing direction (Mitrokhina et al., 2013). The goal is to model two or three artificial images that have the same objects in the field of view that are visible in the original surface image (Lunokhod panorama).
\end{abstract}

In general, most of the features seen in the foreground of panoramas are too small or uneven to be identified in LRO orbital images and DEMs, so at this step we mostly pay attention on the background hills. There we look for several prominent features (mostly relatively fresh craters on the hill slope) and pin their coordinates in order to use them as ground control points on the next step.

The accuracy of the modelled artificial surface image mostly depends on the input DEM. For example, for Lunokhod-2 region DEM with resolution $1.7 \mathrm{~m} / \mathrm{px}$ was obtained (Karachevtseva et al., 2016). Relative position of two points in the DEM can be measured to $0.3-0.5$ px that corresponds to the accuracy of $0.5-0.8 \mathrm{~m}$. On the other hand, pixel size of the surface image depends on camera parameters and the distance to the object. Lunokhod cameras have published focal length 12.5 $\mathrm{mm}$ and pixel size $13.1 \mu \mathrm{m}$, angular resolution of panoramas is $0.06^{\circ}$ (Selivanov et al., 1971). So one pixel of the surface image in the distance of $1.5 \mathrm{~km}$ corresponds to $1.6 \mathrm{~m}$ on the ground. That is twice bigger than accuracy of the selected DEM.

- Joint photogrammetric adjustment of original and synthetic images. On the next step, using photogrammetry software PHOTOMOD (http://www.racurs.ru/) we adjust together original surface image with the modelled ones. For the adjustment we use known exterior orientation for artificial models and preliminary exterior orientation for original surface image. To provide relative orientation we need to measure tiepoints in overlapping areas and ground control points on all images. Then we adjust all the images and measurements in PHOTOMOD and get the position of observation point for the original image.

Challenge here is to find common points between synthetic and real images. There are a lot of panoramas where quality or illumination conditions are not the best, or which capture plain surface without any prominent features (such as boulders, craters, hills on the horizon). The study showed that only about $10 \%$ of Lunokhod-2 panoramas can be used for rover localization. In case of Lunokhod-1 this percentage is even smaller due to the location of the landing site in the Mare far from hills. For selected panoramas usually it is possible to find at first about 5-7 ground control points at the background hills. Tests show that this is enough for the first adjustment and first refinement of exterior orientation for the original image.

Then, using the refined observation coordinates we again model artificial surface image that is now closer to the original one and has more common objects. So the number of control points increases and stabilizes the observation geometry. At first we can identify more objects of the background (on the slopes), and then, after several iterations (adjustment and modelling) we are approaching to the real observation point and it becomes possible to identify also some objects (mostly craters) on a horizontal plane of the foreground. Using such approach we can increase the number of control and tie-points and repeat adjustment until we get the desired accuracy.

To make the identification of control points easier it is possible to model supplementary artificial images from different heights above the surface, e.g. 10-30 m, - on such an image craters on the mare plain are visible better (compare images in Figure 2). As all synthetic images also contain image depth (3D position is known for each pixel) from DEM it is easier to measure tiepoints first between artificial images in horizontal and oblique view and then move the identified points onto the original image.

It is better to collect more than 30 points, however, this number mostly depends on resolution of original surface image and base DEM/ortho, on the angle of the sun, on contrast and texture of surface, on area of the landing site, and experience of the operator.

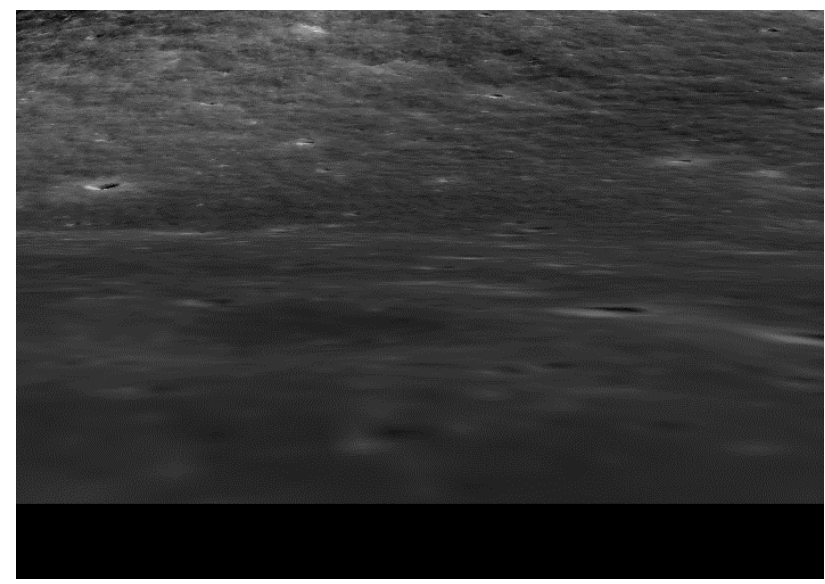




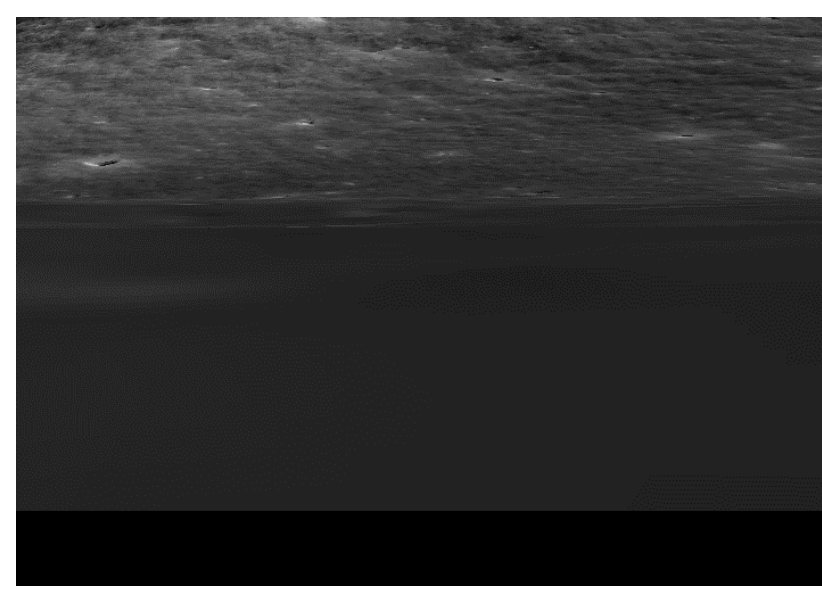

Figure 2. Example of artificial surface image modelling from different observation height: upper - $30 \mathrm{~m}$; lower - $2 \mathrm{~m}$ (Observation coordinates: $25.78469^{\circ} ; 30.80354^{\circ}$, azimuth $190^{\circ}$, downward pointing angle $5^{\circ}$ ).

\section{TEST MEASUREMENTS}

To test the developed technique on original images we selected Lunokhod-2 area. Detailed study of LRO NAC data allowed us to determine Lunokhod rover tracks and refine the route with high accuracy based on created DEM and orthoimage (Karachevtseva et al., 2016). Modern processing of panoramic images (Kozlova et al., 2014) prepared them for further use in this study as original data.

\subsection{Tests on models}

Firstly, we tested this technique on modelled images. In this case we select one of the artificial images to be a "real" image with unknown observation point, carry out the whole procedure of adjustment, and then compare the obtained coordinates with ones used for modelling.

To do this we created three synthetic surface images by means of OrthoDEM2Cam. For modelling we used Lunokhod- 2 region which is close to the highlands and main parameters of Lunokhod panoramic camera (focal length $12.5 \mathrm{~mm}$, pixel size $13.1 \mu \mathrm{m})$. Then we loaded these synthetic images into PHOTOMOD considering two of them as reference images (Model 1 and 2) and the third one as a target (unknown) image with only preliminary exterior orientation (Model 3), see Table 1. A few ground control points were measured in the hills $(\sim 1-1.5 \mathrm{~km}$ far from the selected observation points) corresponding to the most prominent features, which could have been clearly identified in real surface images (Figure 3). On models it is possible to measure plenty of control points, as every pixel from the model corresponds to certain pixel from DEM. However, main attention was paid to find the minimal amount of points which is necessary for first refinement of preliminary orientation for the target image. Bundle-block adjustment of the three models showed that even 5 control points can give sufficient result: the position of observation point was found within the distance of $3.6 \mathrm{~m}$ from the observation point (Table 1).

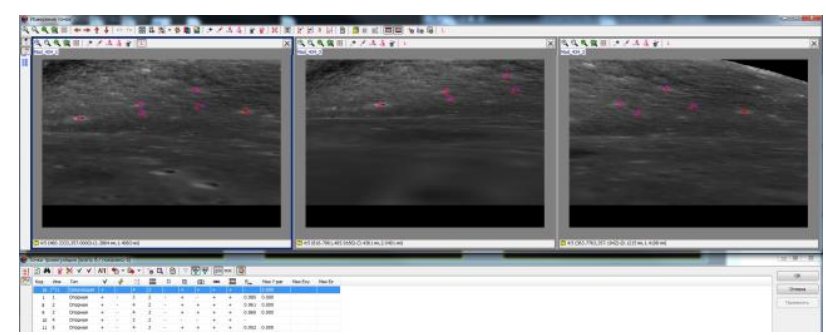

a) measurements of ground control points and tie-points on synthetic images in PHOTOMOD. Target image (Model 3) is in the middle.

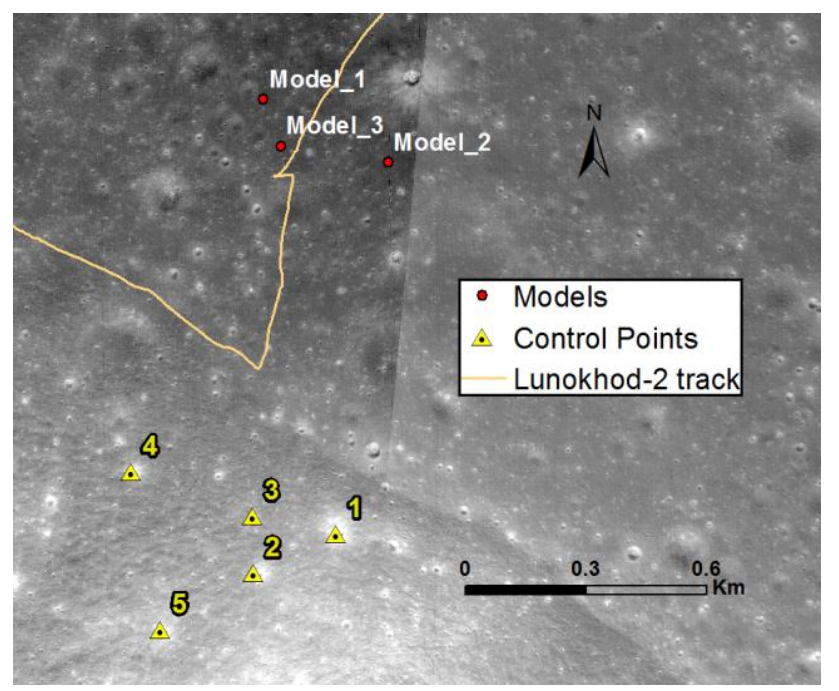

b) positions of ground control and observation points of modelled images. Model 3 is the target (unknown) image.

Figure $3(a, b)$. Example of measurements of ground control points on synthetic images.

Table 1. Exterior orientation parameters of modelled images and results of their adjustment with 5 control points

\begin{tabular}{|l|l|r|r|r|r|r|r|}
\hline \multicolumn{1}{|c|}{ Name } & \multicolumn{1}{|c|}{ Type } & Latitude, $^{\circ}$ & Longitude, $^{\circ}$ & $\mathbf{H}, \mathbf{m}$ & $\boldsymbol{\omega}^{\circ}$ & \multicolumn{1}{c}{$\boldsymbol{}^{\circ}$} & $\mathbf{\kappa}^{\circ}$ \\
\hline Model_1 & reference image & 25.78548 & 30.80124 & -2689.0 & -85.0 & 5.0 & 179.6 \\
\hline Model_2 & reference image & 25.78028 & 30.81264 & -2689.2 & -84.2 & 29.9 & 177.1 \\
\hline Model_3_Pr & $\begin{array}{l}\text { target image (preliminary } \\
\text { coordinates) }\end{array}$ & 25.78321 & 30.80205 & -2690 & -85.0 & 0.3 & 180.0 \\
\hline Model_3_Adj & $\begin{array}{l}\text { target image (adjusted } \\
\text { coordinates) }\end{array}$ & 25.78173 & 30.80275 & -2732.2 & -85.1 & -0.2 & 179.9 \\
\hline & Shift (pr-adj), m & 44.4 & -21.1 & 42.2 & 0.1 & 0.5 & 0.0 \\
\hline & $\begin{array}{l}\text { target image (real } \\
\text { coordinates) }\end{array}$ & 25.78164 & 30.80284 & -2730.2 & -85.0 & 0.0 & 180.0 \\
\hline & Accuracy (adj-real), $\mathbf{m}$ & $\mathbf{2 . 6}$ & $\mathbf{- 2 . 7}$ & $\mathbf{- 2 . 0}$ & $\mathbf{- 0 . 1}$ & $\mathbf{- 0 . 2}$ & $\mathbf{- 0 . 1}$ \\
\hline
\end{tabular}

If we increase number of ground control points up to 15 , we get coordinates with the accuracy of 1-1.5 m.

We expect that adjustment of the real fragment of Lunokhod panorama gives accuracy of the same order (of course, in similar conditions - similar observation geometry).

\subsection{Tests on Lunokhod panoramas}

After tests on models we used as a target image central fragment of real Lunokhod-2 panorama \#434 (Figure 5a), which captures the same part of the slope as Model 3. We measured ground control and tie-points (Figure 4) and carried out adjustment. It is natural that when measuring control and tie-points we can get some errors. While adding points on real Lunokhod images it is easy to make a mistake. However, the technology allows you to 
detect and highlight the outliers as a result of a bundle block adjustment, also due to the number of measurements. Therefore, the more points are measured the more reliable result we obtain. First results gave the position of panorama observation point twice closer to the most probable real location than used preliminary coordinates (see Figure 4), but still about 77 meters away (and 46 meters from the track). Using exterior orientation obtained from adjustment we can remodel the whole panoramic image and compare it with the original one (Figure 5 a,b).

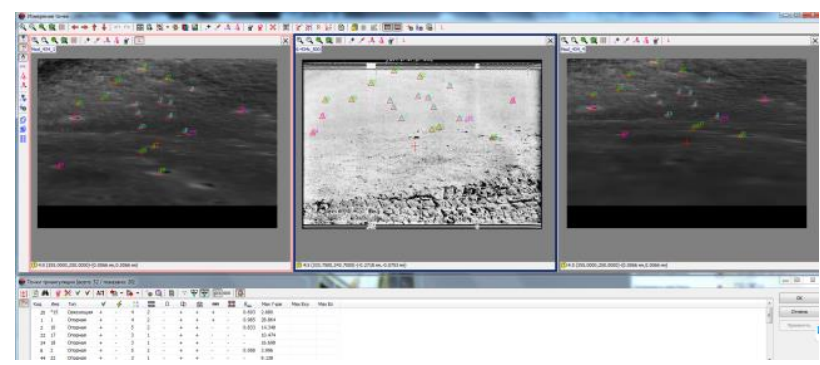

Figure 3. Example of measurements of ground control points and tie-points on original image. Real (unknown) fragment of panorama \#434 is in the middle.

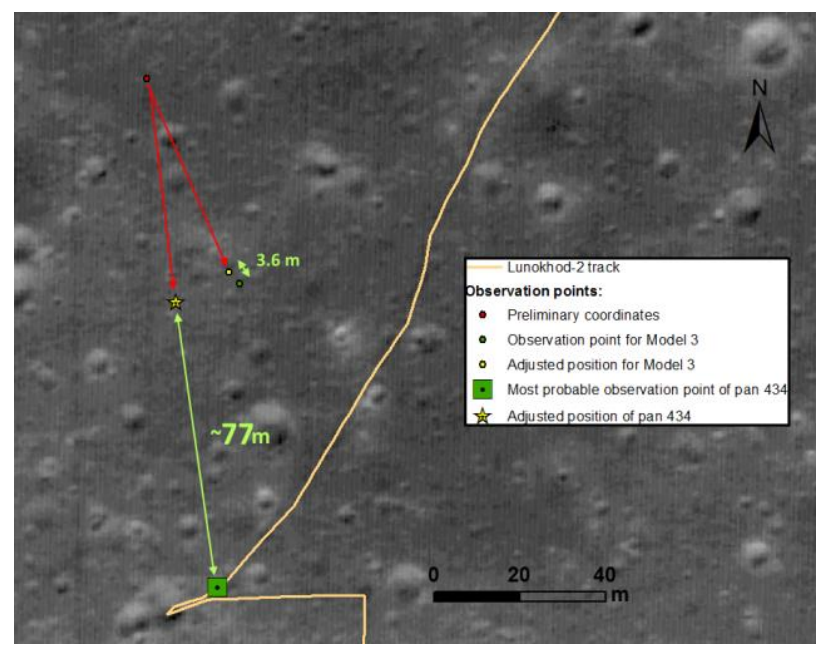

Figure 4. Observation points of Model 3 and panorama \#434: preliminary (red), obtained from adjustment (yellow) and real (green) positions.

We think that such displacement may be caused by the difference between published camera parameters used for modelling and real parameters that cameras had on the lunar surface. So we tried to carry out self-calibration of the camera during adjustment. Distortion of panoramic images was considered to be negligibly small (Rodionov et al., 1973), however, we have obtained corrected parameters of principal point, focal length, and coefficients of distortion - Table 3. Big value of $\mathrm{k} 1$ most probably compensates the difference between spherical projection of original panoramas and central projection used for modelling. Calibration in some examples helped to decrease the distance to the most probable observation point of panorama to $29 \mathrm{~m}$. However, calibration does not give stable result, so we assume that quality of original panoramas and measured control points are not enough for proper calibration. Also these parameters may slightly differ from panorama to panorama due to additional distortion caused while scanning of original films.
Table 3. Calibration of Lunokhod camera

\begin{tabular}{|c|c|}
\hline Parameter & Adjusted values \\
\hline Dx0, mm & -0.0313 \\
\hline Dy0, mm & 0.0400 \\
\hline DF, mm & -0.1618 \\
\hline k1 & -0.002765183 \\
\hline k2 & 0 \\
\hline k3 1 & 0 \\
\hline P2 & 0 \\
\hline
\end{tabular}

\section{CONCLUSION AND FUTURE WORK}

LRO NAC DEM, orthomosaic, and the results of detailed studies of the Lunokhod-2 route were used for modelling of individual sites in order to find observation points for archival panoramas. Recovery of lost elements of exterior orientation for archival images helps to put them into modern geospatial context. This, in turn, makes it possible to carry out detailed geomorphological study of the lunar surface as a result of the new digital processing of archival panoramas.

In the paper we have presented a test approbation of the developed method of determination of rover coordinates from photogrammetry processing of obtained surface images together with artificially modeled synthetic images produced from highresolution DEM and orthomosaic. We found out that the method shows good results on models but needs further tests on real surface images.

In future we plan to test the developed technique on another lunar region using Apollo data (Haase et al., 2012) and discuss the ways of increasing of the accuracy of the method.

\section{REFERENCES}

Haase I., Oberst J., Scholten F. et al. Mapping the Apollo 17 landing site area based on Lunar Reconnaissance Orbiter Camera images and Apollo surface photography // Journal of Geophysical Research, 2012, v. 117, E00H20, doi:10.1029/2011JE003908.

Karachevtseva I., Oberst J., Scholten F. et al., Cartography of the Lunokhod-1 Landing Site and Traverse from LRO Image and Stereo Topographic Data // 2013, Planetary and Space Science, Vol.85, p. 175-187. http://dx.doi.org/10.1016/j.pss.2013.06.002.

Karachevtseva I., Kozlova N., Kokhanov A., Zubarev A., Patratiy V., Konopikhin A., Basilevsky A., Oberst J., Haase I., Joliff B., Plescia J., Robinson M., 2016. Cartography of the Luna-21 landing site and Lunokhod-2 traverse area based on Lunar Reconnaissance Orbiter camera images and surface archive TV-panoramas // Icarus (in press).

Kozlova N., Zubarev A., Karachevtseva I. et al. Some aspects of modern photogrammetric image processing of Soviet Lunokhod panoramas and their implementation for new studies of lunar surface. // The International Archives of the Photogrammetry, Remote Sensing and Spatial Information Sciences, 2014, Volume XL-4, 2014 pp. 121-126. ISPRS Technical Commission IV Symposium, Suzhou, China. doi:10.5194/isprsarchives-XL-4-121-2014. Kozlova_Lunokhod_DOI_panoramas_full_paper.pdf. 
Kozlova N.A., Zubarev A.E., Karachevtseva I.P., Kokhanov A.A., Solodovnikova A.M., Rybakin Y.A. Determination of observation points for lunar surface panoramic images using GIS // Ecology, economics, and computer science. Vol. 3: Geoinformation technologies and space monitoring, 2015, p. 153163 (in Russian).

Mitrokhina L., Zubarev A., Nadezhdina I., Karachevtseva I. Recovery of exterior orientation of Lunokhod panoramic images using LRO terrain models // ISPRS Meeting of the Working Group IV/8 «Advances in Planetary Mapping and Spatial Databases», MIIGAiK, Moscow, Russia, October 9-12, 2013. http://www.miigaik.ru/konf/isprs/.

Rodionov B.N., Nepoklonov B.V., Kiselev V.V., Selivanov A. S., Zasetskiy V.V., Ivanov O.G., Babakov F.I., Mikhailov R.M., Reshetov M.M. Topographical surveys on the lunar surface from the Soviet unmanned spacecraft. 1973, Geodesy and Cartography, № 10. pp. 29-41 (in Russian).

Selivanov A.S., Govorov V.M., Zasetskii V.V., Timohin V.A. Construction properties and main parameters of Lunokhod-1 television systems. In: Mobile laboratory on the Moon
Lunokhod-1. Vol.1. (Ed. Vinogradov A.P.) M.: Nauka, 1971, pp. 55-65 (in Russian).

Zubarev A.E., Nadezhdina I.E., Kozlova N.A., Brusnikin E.S, Kokhanov A.A. and Karachevtseva I.P. Special software for planetary image processing and research // ISPRS Archives 2016

http://www.racurs.ru/

http://www.esri.com

\section{ACKNOWLEDGEMENTS}

The authors are grateful to the reviewers for valuable advice that significantly improved the paper.

This work was carried out at MIIGAiK and supported by Russian Science Foundation, project \#14-22-00197.

Lunokhod panoramic image processing received funding from the European Community's Seventh Framework Program (FP7/2007-2013) under grant agreement № 312377 Planetary Robotics Vision Data Exploitation (PRoViDE).

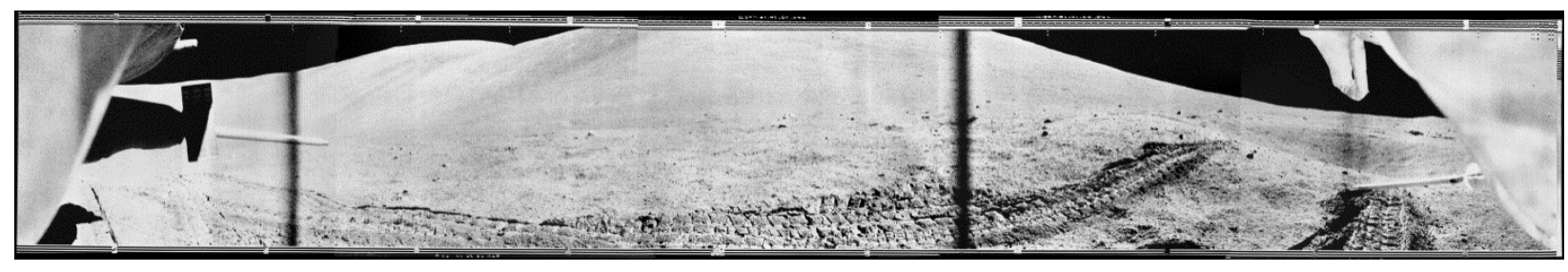

a

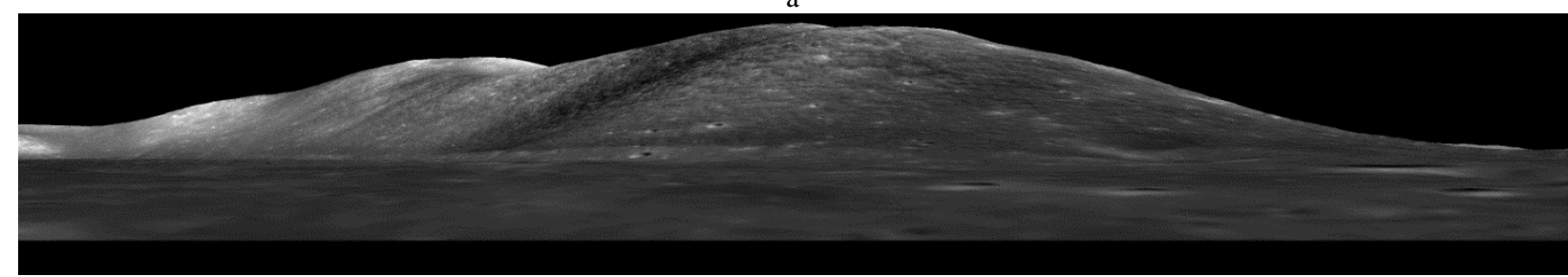

b

Figure 5. a - original Lunokhod-2 panorama \#434; b - synthetically modelled panorama \#434 based on determined exterior orientation. 